INPLASY

PROTOCOL

To cite: Feng et al. The biomechanical properties of barbed suture in human cadaveric model: a systematic review and meta-analysis. Inplasy protocol 202180100. doi:

10.37766/inplasy2021.8.0100

Received: 25 August 2021

Published: 25 August 2021

Corresponding author: Jin Huang

michael_huangjin@163.com

Author Affiliation:

Medical Device Regulatory

Research and Evaluation

Center, Sichuan University.

Support: None.

Review Stage at time of this submission: Preliminary searches.

Conflicts of interest:

None declared.

\section{The biomechanical properties of barbed suture in human cadaveric model: a systematic review and meta-analysis}

Feng, $\mathrm{K}^{1}$; Lin, Y2 ; Huang, J3.

Review question / Objective: P: surgical suturing in a cadaveric model; I: barbed sutures; C: conventional sutures; $O$ : biomechanical properties including stiffness, maximum load, mode of failure, cross-sectional area, etc.

Condition being studied: Suturing is an indispensable procedure of surgery operation. Tenorrhaphy, tendon repair, sacrocolpopexy mesh attachment, arthrotomy closure, skin grafts and abdominal surgery all requires suturing of the incision site. The biomechanical properties of sutures can have a great impact on the suture site. If the suture does not meet its biomechanical requirements, severe complications also known as surgical site infections (SSIs) may occur. SSIs not only lead to an increase in morbidity, readmission, ICU admission and long-term surgical-site complications, but also result in a greater risk of death in patients undergoing surgical procedures.

INPLASY registration number: This protocol was registered with the International Platform of Registered Systematic Review and Meta-Analysis Protocols (INPLASY) on 25 August 2021 and was last updated on 25 August 2021 (registration number INPLASY202180100).

\section{INTRODUCTION}

Review question / Objective: P: surgical suturing in a cadaveric model; I: barbed sutures; $C$ : conventional sutures; 0 : biomechanical properties including stiffness, maximum load, mode of failure, cross-sectional area, etc.
Rationale: Suturing is widely used in all kinds of surgery. Many kinds of sutures have been invented to adapt to the different requirements from variable surgeries these years. Still, there remain many pending issues and limitations regarding the usage of different sutures, including severe complications. The biomechanical properties of sutures in human anatomy is 
of vital importance. Research regarding this field still remains vacant.

Condition being studied: Suturing is an indispensable procedure of surgery operation. Tenorrhaphy, tendon repair, sacrocolpopexy mesh attachment, arthrotomy closure, skin grafts and abdominal surgery all requires suturing of the incision site. The biomechanical properties of sutures can have a great impact on the suture site. If the suture does not meet its biomechanical requirements, severe complications also known as surgical site infections (SSIs) may occur. SSIs not only lead to an increase in morbidity, readmission, ICU admission and long-term surgical-site complications, but also result in a greater risk of death in patients undergoing surgical procedures.

\section{METHODS}

Participant or population: Human cadaver tissue.

Intervention: Wound being anastomose by conventional suture.

Comparator: Wound being anastomose by barbed suture.

Study designs to be included: Humancadaveric experiments.

Eligibility criteria: The inclusion criteria were as follows: (a) study design had to be a RCT carried out on a human cadaveric model; (b) the control group and the intervention group have to be conventional suturing vs. barbed suturing; (c) at least one of the results of the study had to be clearly stated in detail data. The exclusion criteria were as follows: (a) the control and intervention group were other materials, such as staple or suture anchor; (b) experiments carried out on animal model; (c) study design was not RCT, such as case report or systematic review.

Information sources: The MEDLINE, EMBASE and the Cochrane Library databases were searched using the following term: "cadaver" OR "cadaveric"
OR "corpse" OR "dead body" AND "barbed" OR "knotless" AND "suture" OR "suturing" (last updated in 10th, Aug 2021).

Main outcome(s): Repair sites' load to failure, stiffness, mode of failure.

Additional outcome(s): Suturing time, visual analog scale.

Quality assessment / Risk of bias analysis: To evaluate the ROB in each study, we use the Anatomical Quality Assurance (AQUA) checklist. The checklist was divided into 5 domains and composed of 29 specific criteria.

Strategy of data synthesis: The studies were divided into groups according to the method applied in each of the experiments. The distraction of the repair site testing its tensile load is commonly used in flexor tendon repairs, thus, a meta-analysis was performed to combine those data. Subgroup analysis was performed regarding to different characteristics of each experiment. Statistical analyses were carried out using Review Manager (Version 5.4.1 ). We estimated the pooled mean difference (MD) in continuous data and the risk ratios (RR) in dichotomous data using the random effect model. The heterogeneity between studies was calculated via the $X 2$ test and 12 test. A pvalue of less than 0.05 was considered to indicate a significant statistical difference.

Subgroup analysis: Subgroup analysis will be carried out according to the different methods applied in each of the experiments included.

Sensitivity analysis: Sensitivity analysis will be carried out using Review Manager (Version 5.4.1 ). Sensitivity will be carried out based on the different methods applied in each of the experiments included.

Language: No restriction.

Country(ies) involved: China.

Other relevant information: There is no conflict of interest. This research did not 
receive any specific grant from funding agencies in the public, commercial, or notfor-profit sectors.

Keywords: barbed suture; cadaveric; biomechanical properties; tendon repair.

Contributions of each author:

Author 1 - Kun Feng.

Email: sam.fkun@gmail.com

Author 2 - Yifei Lin.

Author 3 - Jin Huang.

Email: michael_huangjin@163.com 